\title{
Dietary patterns modify the association between fat mass and obesity-associated genetic variants and changes in obesity phenotypes
}

\author{
Firoozeh Hosseini-Esfahani $^{1}$, Glareh Koochakpoor ${ }^{2}$, Parvin Mirmiran ${ }^{1,3 *}$, Maryam S. Daneshpour ${ }^{4 *}$ \\ and Fereidoun Azizi ${ }^{5}$ \\ ${ }^{1}$ Nutrition and Endocrine Research Center, Research Institute for Endocrine Sciences, Shabid Beheshti University \\ of Medical Sciences, Tebran, 1985717413, Iran \\ ${ }^{2}$ Maragheh University of Medical Sciences, Maragheh, 9415969788, Iran \\ ${ }^{3}$ Faculty of Nutrition Sciences and Food Technology, National Nutrition and Food Technology Research Institute, \\ Shahid Beheshti University of Medical Sciences, Tehran, 1981619573, Iran \\ ${ }^{4}$ Cellular and Molecular Endocrine Research Center, Research Institute for Endocrine Sciences, Shabid Beheshti \\ University of Medical Sciences, Tehran, 1985717413, Iran \\ ${ }^{5}$ Endocrine Research Center, Research Institute for Endocrine Sciences, Shahid Beheshti University of Medical Sciences, \\ Tehran, 1985717413, Iran
}

(Submitted 26 June 2018 - Final revision received 3 December 2018 - Accepted 6 February 2019 - First published online 31 May 2019 )

\begin{abstract}
The present study investigated whether dietary patterns could interact with fat mass and obesity-associated (FTO) polymorphisms in relation to changes in BMI and waist circumference (WC) over 3.6 years of follow-up. Subjects were selected from participants of the Tehran Lipid and Glucose Study ( $n$ 4292, 43.2 \% male). Dietary data were collected using a valid and reliable FFQ. Dietary patterns were determined using factor analysis. The genotypes of polymorphisms (rs1421085, rs1121980, rs17817449, rs8050136, rs9939973 and rs3751812) were determined. Genetic risk score (GRS) was calculated using the weighted method. Mean ages of men and women were $42 \cdot 6$ (SD 14) and 40.4 (SD 13) years, respectively. The healthy (e.g. vegetables and fruits) and the Western dietary patterns (WDP; e.g. soft drinks and fast foods) were extracted. In carriers of the risk alleles rs1121980, rs1421085, rs8050136, rs1781799 and rs3751812, BMI was approximately 2-fold higher in individuals in the higher quartile of WDP score, compared with the first quartile $(P<0 \cdot 05)$. WC increased with increasing WDP score in carriers of the risk alleles rs1121980 and rs3751812, but not in individuals who did not carry any risk alleles. BMI and WC increased to a greater extent in the high GRS group while increasing quartiles of the WDP score, compared with the low GRS group (BMI change; Q1: 1.04 (SE 0.34) $v$. Q4: 2.26 (SE 0.36)) (WC change; Q1: $0.47\left(\mathrm{se} \mathrm{0.32)v.} \mathrm{Q4:} \mathrm{0.95} \mathrm{(sE} \mathrm{0.34))} \mathrm{(} P_{\text {interaction }}<0.05\right)$. These results suggest that adults with higher genetic predisposition to obesity are more susceptible to the harmful effects of adherence to the WDP, which emphasised the need to reduce the consumption of unhealthy foods for the prevention of obesity.
\end{abstract}

Key words: FTO polymorphisms: Dietary patterns: Obesity: Interactions

Obesity, as a serious risk factor for non-communicable diseases, is the fifth leading cause of death worldwide ${ }^{(1)}$, with statistics dramatically increasing globally ${ }^{(2)}$, emphasising the urgency in the development of strategies for obesity prevention in recent years $^{(3)}$.

In order to develop an appropriate strategy to prevent obesity, factors contributing to obesity should be first considered. It is well recognised that both genetic and environmental factors contribute to the development of obesity ${ }^{(4)}$. Changes in lifestyle factors such as improving dietary patterns are key elements of obesity prevention policies; however, the success of their implementation may be affected by genetic susceptibility ${ }^{(5,6)}$. Therefore, understanding the interactions between genetic background and nutritional factors could be helpful in developing a costeffective strategy for the prevention of obesity.

Polymorphisms of the fat mass and obesity-associated (FTO) gene, as the strongest genetic determinant of obesity, increase appetite and consequently increase the risk of obesity (1.67 fold $)^{(7)}$. Interactions between polymorphisms of the FTO gene and nutrient intake in relation to obesity have only been

Abbreviations: FCT, food composition table; FTO, fat mass and obesity-associated; GRS, genetic risk score; WC, waist circumference.

* Corresponding authors: Parvin Mirmiran, email mirmiran@endocrine.ac.ir; Maryam S. Daneshpour, email Daneshpour@endocrine.ac.ir 
examined in few studies ${ }^{(8,9)}$, none of which specifically examined the modulatory effect of dietary patterns on the associations of the FTO risk alleles with obesity. Single foods or nutrients cannot predict obesity development, while dietary patterns are significantly related to obesity over time ${ }^{(10,11)}$, so the measurement of dietary patterns has been recommended to investigate the links between dietary factors and obesity. The aim of the current study was to evaluate whether the relationship between different dietary patterns and changes in BMI and waist circumference (WC) differed according to FTO genotypes of adult individuals. Identifying these environmental interactions is essential for implementing appropriate preventive approaches in individuals with greater genetic susceptibility to obesity.

\section{Materials and Methods}

\section{Subjects}

Subjects of this cohort study were selected from among Tehran Lipid and Glucose Study participants ${ }^{(12,13)}$, a population-based prospective study performed to determine risk factors for noncommunicable diseases in a sample of residents of District 13 of Tehran, the capital of Iran. The first examination survey was performed from 1999 to 2001 on 15005 individuals, aged $\geq 3$ years, and follow-up examinations were conducted every 3 years (2002-2005; 2005-2008; 2008-2011 and 2011-2014) to identify recently developed diseases.

For the present study, of 12823 participants who entered in the fourth survey (2008-2011), 7812 were randomly selected for dietary assessment based on age and sex; of these, 6874 adult participants aged $\geq 18$ years were included and followed. Subjects who did not complete anthropometric data at baseline and follow-up survey ( $n$ 920), were without DNA sample or lacking DNA purification ( $n$ 1182), were without accurate report of energy intake (whose reported energy intake divided by the predicted energy intake did not meet the $\pm 3 \mathrm{SD}$ range), were pregnant or breast-feeding women and those taking drugs that affect weight were excluded ( $n$ 480). Finally, 4292 individuals, with a mean 3.6 years of follow-up, remained in the study.

\section{Measurements}

Usual dietary intake was assessed using a valid and reliable 168-item semi-quantitative $\mathrm{FFQ}^{(14,15)}$, which comprised a list of foods with standard serving sizes; the portion sizes were converted to $\mathrm{g} / \mathrm{d}$ using household measures. Based on the nutrient composition of food items and available literature, eighteen food groups were classified. Since the Iranian food composition table (FCT) is incomplete ${ }^{(16)}$, the United States Department of Agriculture FCT was used to analyse foods ${ }^{(17)}$. However for some national foods and beverages which were not listed in the United States Department of Agriculture FCT, the Iranian FCT was used.

Body weight was measured to the nearest $100 \mathrm{~g}$ using digital scales (model 707; Seca). Height was measured to the nearest $0.5 \mathrm{~cm}$ using stadiometer (model 208 Portable Body Meter Measuring Device; Seca). WC was measured and recorded to the nearest $0 \cdot 1 \mathrm{~cm}$ using a non-stretch tape measure. BMI $\left(\mathrm{kg} / \mathrm{m}^{2}\right)$ and WC $(\mathrm{cm})$ change were calculated by subtracting the BMI and WC at baseline from their measurements at follow-up; increases in BMI and WC were defined if BMI or WC change was positive or $>0$.

The physical activity level was assessed with high reliability and relatively moderate validity using the Persian translated modifiable activity questionnaire ${ }^{(18)}$. Frequency and time spent on light, moderate, hard and very hard intensity activities, according to the list of common activities of daily life over the past year, were obtained, and these activity data were converted into metabolic equivalent/hours/week.

\section{Genotyping}

Six SNP were selected (rs1421085, rs1121980, rs17817449, rs8050136, rs9939973 and rs3751812) within the region of the FTO gene, based on the literature available and on the validated catalogue of published genome-wide association studies and the Phenotype-Genotype Integrator ${ }^{(19,20)}$, taking into account minor allele frequency $>0.2$ and $P$ values $<10^{-7}$. The selected SNP were associated with dietary intake or obesity phenotypes ${ }^{(21-26)}$. Genomic DNA was extracted from peripheral blood $^{(27)}$. Portions of DNA samples were genotyped with HumanOmniExpress-24-v1-0 bead chips, containing 649932 SNP loci, with an average mean distance of $4 \mathrm{~kb}$ at the deCODE genetics company (Reykjavik, Iceland) according the manufacturer's specifications (Illumina Inc.). For quality control procedures, PLINK programme (v. 1.07) and R statistic (v. 3.2) were used with the total genotyping rate of 0.9774 . After quality control procedures, the genotyping data of FTO polymorphisms were used for data analysis ${ }^{(28)}$.

\section{Obesity genetic risk score calculation}

Genetic risk score (GRS) was calculated based on the six SNP using the weighted method ${ }^{(29,30)}$. Each SNP was reported as 0,1 or 2 based on the number of risk alleles (BMI-increasing alleles) ${ }^{(19,20)}$, and each SNP was weighted by its relative effect size (OR), derived from previously reported meta-analyses or original data (Table 1$)^{(21,24,31)}$. We then calculated the GRS using the following equation:

$$
\begin{aligned}
\mathrm{GRS}= & (\mathrm{OR} 1 \times \mathrm{SNP} 1+\mathrm{OR} 2 \times \mathrm{SNP} 2+\ldots+\mathrm{OR} n \times \mathrm{SNP} n) \\
& \times(n / \text { sum of the OR })
\end{aligned}
$$

OR is the OR of each individual SNP on BMI, $n$ is 6 and sum of the OR is 8.18 in the current analysis. The GRS ranged from 0 to 12 , and each point of the GRS conformed to each single risk allele.

\section{Statistical analysis}

Statistical analyses were carried out using the Statistical Package for Social Sciences (version 21.0). A $P$ value $<0.05$ was used to determine statistical significance. The descriptive analysis consisted of a comparison of qualitative and quantitative variables across quartiles of dietary pattern scores using the chi square and ANOVA test, respectively; genotype and allele frequencies for the analysed polymorphisms were calculated. Pearson's $\chi^{2}$ 
Table 1. Genotype information of fat mass and obesity-associated (FTO) polymorphisms among the population of the Tehran Lipid and Glucose Study*

\begin{tabular}{|c|c|c|c|c|c|}
\hline Polymorphisms & & Frequency & Risk allele & HWE $P$ & Effect size \\
\hline rs1121980 & & & $\mathrm{T}$ & 0.89 & 1.34 \\
\hline \multirow[t]{2}{*}{ Allele } & C & 59.0 & & & \\
\hline & $\mathrm{T}$ & $41 \cdot 0$ & & & \\
\hline \multirow[t]{3}{*}{ Genotype } & $\mathrm{CC}$ & $37 \cdot 4$ & & & \\
\hline & CT & 47.5 & & & \\
\hline & TT & $15 \cdot 1$ & & & \\
\hline rs1421085 & & & C & 0.87 & 1.43 \\
\hline \multirow[t]{2}{*}{ Allele } & $\mathrm{T}$ & $62 \cdot 0$ & & & \\
\hline & C & 38.0 & & & \\
\hline \multirow[t]{3}{*}{ Genotype } & $\mathrm{TT}$ & 38.9 & & & \\
\hline & $\mathrm{TC}$ & $47 \cdot 2$ & & & \\
\hline & $\mathrm{CC}$ & 13.9 & & & \\
\hline rs9939973 & & & A & 0.87 & $1 \cdot 10$ \\
\hline \multirow[t]{2}{*}{ Allele } & G & $61 \cdot 0$ & & & \\
\hline & A & $39 \cdot 0$ & & & \\
\hline \multirow[t]{3}{*}{ Genotype } & GG & 37.5 & & & \\
\hline & GA & $47 \cdot 6$ & & & \\
\hline & AA & 14.9 & & & \\
\hline rs8050136 & & & A & 0.96 & 1.25 \\
\hline \multirow[t]{2}{*}{ Allele } & C & $64 \cdot 0$ & & & \\
\hline & A & $36 \cdot 0$ & & & \\
\hline \multirow[t]{3}{*}{ Genotype } & $\mathrm{CC}$ & $13 \cdot 9$ & & & \\
\hline & $\mathrm{CA}$ & $45 \cdot 4$ & & & \\
\hline & AA & $40 \cdot 7$ & & & \\
\hline rs17817449 & & & G & 0.93 & 1.54 \\
\hline \multirow[t]{2}{*}{ Allele } & $\mathrm{T}$ & $64 \cdot 0$ & & & \\
\hline & $\mathrm{G}$ & $36 \cdot 0$ & & & \\
\hline \multirow[t]{3}{*}{ Genotype } & TT & $41 \cdot 8$ & & & \\
\hline & TG & $45 \cdot 7$ & & & \\
\hline & GG & $12 \cdot 4$ & & & \\
\hline rs3751812 & & & $\mathrm{T}$ & 0.97 & 1.52 \\
\hline \multirow[t]{2}{*}{ Allele frequency } & G & $65 \cdot 0$ & & & \\
\hline & $T$ & $35 \cdot 0$ & & & \\
\hline \multirow[t]{3}{*}{ Genotype } & GG & $43 \cdot 0$ & & & \\
\hline & GT & 44.9 & & & \\
\hline & $\mathrm{TT}$ & $12 \cdot 0$ & & & \\
\hline
\end{tabular}

HWE, Hardy-Weinberg equilibrium.

* Risk alleles were based on data from the GWAS Catalog (the NHGRI-EBI Catalog of published genome-wide association studies ${ }^{(19)}$ ) and the Phenotype-Genotype Integrator. Effect sizes were derived from previous literature and reported meta-analyses.

statistic test was used to calculate the Hardy-Weinberg equilibrium.

Dietary patterns were determined using principal component analysis with varimax rotation based on eighteen energy-adjusted food groups $(\mathrm{g} / \mathrm{d})$. Two factors were maintained based on the interpretability of the factors and the scree plot. Factor loadings below $\pm 0 \cdot 2$ are not reported because of the low contribution of their food groups explained by the factor and for the easy explanation of these dietary patterns. Factor scores of each subject were computed by the sum of multiplying the consumption of food groups by their factor loadings on each pattern. Each subject with a higher score on a particular factor had a higher comparative adherence to this pattern compared with others. Participants were separated into two groups based on the median GRS ( $\geq$ median and $<$ median).

To test the interaction between dietary patterns and FTO gene variants or their GRS in relation to changes in BMI and WC, general linear models, including the corresponding main effects
Table 2. Factor loadings for the two dietary patterns identified in study participants

\begin{tabular}{lcr}
\hline & \multicolumn{2}{c}{ Dietary patterns } \\
\cline { 2 - 3 } Food groups & Western & Healthy \\
\hline Sweets and sugar & $0.61^{*}$ & \\
Soft drinks & 0.68 & \\
Red meat & 0.31 & \\
Organ meat & 0.44 & \\
Solid fats & 0.48 & \\
Refined grains & 0.42 & 0.31 \\
Fast foods & 0.59 & \\
Salty snacks & 0.38 & 0.38 \\
High-fat dairy products & 0.34 & 0.70 \\
Fish and poultry & & 0.51 \\
Vegetables & & 0.44 \\
Legumes & & 0.45 \\
Fruit juice & 0.32 & 0.28 \\
Low-fat dairy products & & 0.31 \\
Whole grains & & 0.58 \\
Liquid oil & & 0.20 \\
Fruits & & 7.5 \\
Nuts and seeds & 15.7 & \\
Variance (\%)† & & \\
\hline
\end{tabular}

* Values are factor loadings of dietary patterns measured by factor analysis. Factor loadings below \pm 0.2 are not shown in the table for simplicity.

† Eigenvalues $>1$, Kaiser-Meyer-Olkin (KMO): 0.77.

and interaction terms, were used. Subjects were divided into eight groups based on their combined role of quartiles of dietary patterns and dominant model of FTO SNP genotypes or $\geq$ median and $<$ median GRS in estimating mean and standard error changes of BMI and WC. Models were adjusted for sex, age, education levels ( $>14$ and $\leq 14$ years), smoking (never smoked, ex-smoker and current smoker), baseline physical activity (light, moderate, high), BMI and energy intake. The $P$ value for trend across the quartiles of dietary patterns was determined using logistic regression, with the median of each quartile of dietary patterns as a continuous variable.

\section{Results}

\section{Genotype information of study participants}

Mean ages of 4608 participants (56.8\% women) were $42 \cdot 6$ (sD 14) and 40.4 (sD 13) years in men and women, respectively. Frequency of genotypes or alleles, the effect size and risk allele of each SNP for obesity traits are shown in Table 1. Genotype frequencies did not deviate from Hardy-Weinberg equilibrium.

\section{Identified dietary patterns}

Two major dietary patterns were identified; the healthy dietary pattern included high levels of vegetables, fruits and fruit juices, fish and poultry, legumes, nuts and seeds, low-fat dairy products, whole grains and liquid oil, whereas the Western dietary pattern included high intake of soft drinks, fast foods, sweets and sugar, solid oil, red meat, organ meat, salty snacks, refined grains and high-fat dairy products (Table 2 ). 
Table 3. Characteristics of the study population according to dietary pattern scores among adult participants of the Tehran Lipid and Glucose Study ( $n$ 4292) (Mean values and standard deviations; percentages)

\begin{tabular}{|c|c|c|c|c|c|c|c|c|c|c|c|c|c|c|c|c|c|c|}
\hline & \multicolumn{9}{|c|}{ Healthy dietary pattern } & \multicolumn{9}{|c|}{ Western dietary pattern } \\
\hline & \multicolumn{2}{|c|}{ Q1 } & \multicolumn{2}{|c|}{ Q2 } & \multicolumn{2}{|c|}{ Q3 } & \multicolumn{2}{|c|}{ Q4 } & \multirow[b]{2}{*}{$P$} & \multicolumn{2}{|c|}{ Q1 } & \multicolumn{2}{|c|}{ Q2 } & \multicolumn{2}{|c|}{ Q3 } & \multicolumn{2}{|c|}{ Q4 } & \multirow[b]{2}{*}{$P$} \\
\hline & Mean & SD & Mean & SD & Mean & SD & Mean & SD & & Mean & SD & Mean & SD & Mean & SD & Mean & SD & \\
\hline Sex (female \%) & \multicolumn{2}{|c|}{$50 \cdot 4$} & \multicolumn{2}{|c|}{$55 \cdot 7$} & \multicolumn{2}{|c|}{$57 \cdot 0$} & \multicolumn{2}{|c|}{63.9} & $<0.01$ & \multicolumn{2}{|c|}{$63 \cdot 8$} & \multicolumn{2}{|c|}{$61 \cdot 2$} & \multicolumn{2}{|c|}{$54 \cdot 3$} & \multicolumn{2}{|c|}{$43 \cdot 2$} & $<0.01$ \\
\hline Age (years) & $38 \cdot 6^{*}$ & $13 \cdot 4$ & $41 \cdot 0$ & $13 \cdot 9$ & $42 \cdot 4$ & $14 \cdot 0$ & $42 \cdot 1$ & $14 \cdot 0$ & $<0.01$ & $48 \cdot 1$ & $13 \cdot 6$ & $42 \cdot 1$ & $13 \cdot 2$ & 39.1 & $12 \cdot 8$ & 34.9 & $12 \cdot 2$ & $<0.01$ \\
\hline Current smoker (\%) & \multicolumn{2}{|c|}{$15 \cdot 5$} & \multicolumn{2}{|c|}{$16 \cdot 6$} & \multicolumn{2}{|c|}{$16 \cdot 2$} & \multicolumn{2}{|c|}{$13 \cdot 1$} & 0.09 & \multicolumn{2}{|c|}{10.7} & \multicolumn{2}{|c|}{13.9} & \multicolumn{2}{|c|}{$15 \cdot 8$} & \multicolumn{2}{|c|}{$21 \cdot 1$} & $<0.01$ \\
\hline $\begin{array}{l}\text { Physical activity } \\
\text { (MET/min per week) }\end{array}$ & 474 & 810 & 539 & 841 & 614 & 897 & 707 & 970 & $<0.01$ & 627 & 796 & 522 & 761 & 527 & 797 & 659 & 1130 & \\
\hline Education level $(\%)^{\star}$ & \multicolumn{2}{|c|}{$36 \cdot 4$} & \multicolumn{2}{|c|}{$34 \cdot 0$} & 32 & & 35 & 4 & 0.15 & 26 . & & 32. & & 35 & & 43 & & $<0.01$ \\
\hline Baseline BMI $\left(\mathrm{kg} / \mathrm{m}^{2}\right)$ & $26 \cdot 6$ & 4.91 & $27 \cdot 2$ & 4.69 & $27 \cdot 4$ & 4.57 & $28 \cdot 1$ & 4.87 & $<0.01$ & $28 \cdot 2$ & $4 \cdot 81$ & $27 \cdot 3$ & 4.55 & $27 \cdot 0$ & 4.69 & $26 \cdot 9$ & 4.99 & $<0.01$ \\
\hline BMI change $\left(\mathrm{kg} / \mathrm{m}^{2}\right)$ & $1 \cdot 85$ & $7 \cdot 54$ & 1.64 & $7 \cdot 82$ & 1.59 & $7 \cdot 59$ & 1.64 & $7 \cdot 04$ & 0.84 & 0.50 & $6 \cdot 79$ & 1.68 & 7.98 & 1.95 & $7 \cdot 07$ & $2 \cdot 57$ & 7.94 & $<0.01$ \\
\hline $\begin{array}{l}\text { Baseline waist } \\
\text { circumference }(\mathrm{cm})\end{array}$ & $90 \cdot 7$ & $12 \cdot 4$ & $92 \cdot 1$ & $12 \cdot 1$ & $93 \cdot 0$ & $11 \cdot 8$ & $93 \cdot 8$ & $12 \cdot 2$ & $<0.01$ & $94 \cdot 0$ & 11.4 & $92 \cdot 1$ & $11 \cdot 6$ & 91.5 & $12 \cdot 4$ & $92 \cdot 0$ & $13 \cdot 0$ & $<0.01$ \\
\hline $\begin{array}{l}\text { Waist circumference } \\
\text { change }(\mathrm{cm})\end{array}$ & 1.02 & 7.03 & 0.68 & $7 \cdot 26$ & 0.91 & $7 \cdot 35$ & 0.67 & $7 \cdot 05$ & 0.58 & 0.21 & $7 \cdot 37$ & 0.63 & $7 \cdot 66$ & 1.06 & $7 \cdot 11$ & 1.39 & $6 \cdot 44$ & $<0.01$ \\
\hline Energy intake (kcal)† & 82 & 713 & 2294 & 788 & 2608 & 870 & 3443 & 1290 & $<0.01$ & 2004 & 732 & 2265 & 787 & 2623 & 837 & 3435 & 1311 & $<0.01$ \\
\hline $\mathrm{CH}$ & $3 \cdot 3$ & 6.93 & 3.4 & $6 \cdot 50$ & 58.9 & $6 \cdot 84$ & & $7 \cdot 6$ & & $61 \cdot 0$ & $6 \cdot 66$ & $59 \cdot 2$ & $7 \cdot 11$ & $58 \cdot 0$ & $6 \cdot 69$ & $56 \cdot 8$ & & $<0.01$ \\
\hline $\begin{array}{l}\text { Protein intake } \\
\text { (\% of energy) }\end{array}$ & $14 \cdot 1$ & $2 \cdot 84$ & $14 \cdot 8$ & $2 \cdot 73$ & $15 \cdot 1$ & 3.39 & $15 \cdot 4$ & $3 \cdot 11$ & $<0.01$ & $15 \cdot 9$ & 3.59 & $15 \cdot 0$ & 3.02 & $14 \cdot 4$ & 2.67 & $14 \cdot 1$ & 2.58 & $<0.01$ \\
\hline Total fat (\% of energy) & 0.4 & $7 \cdot 16$ & 0.2 & $6 \cdot 33$ & 29.9 & $6 \cdot 02$ & $29 \cdot 6$ & 6.52 & & 27.5 & 5.72 & $29 \cdot 7$ & $6 \cdot 26$ & $30 \cdot 8$ & 6.59 & $32 \cdot 1$ & 6.63 & $<0.01$ \\
\hline $\begin{array}{l}\text { Fibre intake }(g / 1000 \text { kcal } \\
(4184 \mathrm{~kJ}))\end{array}$ & $7 \cdot 66$ & $2 \cdot 07$ & $9 \cdot 48$ & $2 \cdot 42$ & $10 \cdot 62$ & $2 \cdot 83$ & $12 \cdot 86$ & $4 \cdot 61$ & $<0.01$ & $11 \cdot 87$ & $3 \cdot 82$ & $10 \cdot 37$ & 3.25 & 9.46 & $2 \cdot 97$ & 8.91 & $3 \cdot 83$ & $<0.01$ \\
\hline $\begin{array}{l}\text { Saturated fat } \\
\text { (\% of energy) }\end{array}$ & $10 \cdot 17$ & 3.08 & 9.93 & $2 \cdot 67$ & $9 \cdot 58$ & $2 \cdot 48$ & $9 \cdot 21$ & $2 \cdot 83$ & $<0.01$ & 9.06 & $2 \cdot 54$ & $9 \cdot 64$ & $2 \cdot 70$ & 9.98 & $2 \cdot 93$ & $10 \cdot 22$ & $2 \cdot 87$ & $<0.01$ \\
\hline MUFA (\% of energy) & 0.32 & 2.92 & $10 \cdot 01$ & 2.55 & 9.87 & 2.93 & 9.57 & $2 \cdot 7$ & & $9 \cdot 12$ & 2.38 & 9.90 & $3 \cdot 22$ & $10 \cdot 17$ & $2 \cdot 61$ & 10.57 & 2.71 & $<0.01$ \\
\hline PUFA ( $\%$ of energy) & $6 \cdot 27$ & 2.69 & 6.03 & $2 \cdot 50$ & 5.99 & $2 \cdot 26$ & 5.83 & 2.04 & $<0.01$ & 5.46 & 2.32 & 5.91 & $2 \cdot 24$ & $6 \cdot 26$ & 2.59 & 6.49 & 2.27 & $<0.01$ \\
\hline
\end{tabular}

$\mathrm{Q}$, quartiles of dietary pattern scores, $\mathrm{MET}$, metabolic equivalent, $\mathrm{CHO}$, carbohydrate.

* Educational level $\geq 14$ years.

† To convert energy in kcal to $\mathrm{kJ}$, multiply by $4 \cdot 184$.

\section{Characteristics of participants based on dietary pattern scores}

Table 3 shows baseline general characteristics and dietary intake of participants across dietary pattern scores. Individuals with higher adherence to the Western dietary pattern were younger, while with increasing adherence to the healthy dietary pattern, the age of participants significantly increased.

Subjects with higher score of the Western dietary pattern were more likely to be men and current smokers and have an education level $\geq 14$ years, compared with subjects with higher score of the healthy dietary pattern who were more likely to be women and were more physically active. Individuals with higher adherence to the Western dietary pattern had lower BMI and WC at baseline, compared with participants with lower adherence. Higher adherence to the Western dietary pattern was associated with greater increments in BMI and WC after follow-up years, which is in contrary to subjects with higher adherence to the healthy dietary patterns.

Energy intake was higher in the highest quartile of both dietary pattern scores. There were significantly higher percentages of energy from all kinds of fats in higher categories of the Western dietary pattern. In addition, the percentage of energy from carbohydrate and fibre intake dropped significantly moving from the first to the last category of the Western dietary pattern. Fibre, carbohydrate and protein intake increased across quartiles of the healthy dietary pattern. The percentage of energy from all kinds of fats decreased in higher categories of the healthy dietary pattern.
Changes in BMI according to quartiles of dietary pattern scores by fat mass and obesity-associated genotypes and genetic risk score

Changes in BMI according to quartiles of dietary pattern scores by FTO genotypes are shown in Table 4. The interaction term did not reach statistical significance between FTO polymorphisms or their GRS and the healthy dietary pattern score in relation to BMI change. However, bigger changes (approximately2fold increase) in BMI were observed for risk allele carriers of rs1121980, rs1421085, rs8050136, rs1781799 and rs3751812 with increasing quartiles of the Western dietary pattern $(P<0.05)$. The increase in the Western dietary pattern score was associated with the increase in BMI, an association more noticeable in subjects with high GRS, compared with subjects with low GRS $\left(P_{\text {interaction }}=0 \cdot 01\right)$.

Changes in waist circumference according to quartiles of dietary pattern scores by fat mass and obesity-associated genotypes and genetic risk score

Changes in WC according to quartiles of dietary pattern scores by FTO genotypes or their GRS are shown in Table 5. There were statistically significant interaction between rs1421085, rs3751812 and the Western dietary pattern score in relation to WC change $\left(P_{\text {interaction }}=0.04\right)$.

There was a statistically significant interaction between the GRS and Western dietary pattern score in relation to change in WC. WC increased to a greater extent in high GRS group while 
Table 4. Changes in BMI $\left(\mathrm{kg} / \mathrm{m}^{2}\right)$ according to quartiles of dietary pattern scores (Q) by fat mass and obesity-associated (FTO) genotypes and genetic risk score (GRS) in adult participants of the Tehran Lipid and Glucose Study*

(Mean values with their standard errors)

\begin{tabular}{|c|c|c|c|c|c|c|c|c|c|c|c|c|c|c|c|c|c|c|c|c|}
\hline & \multicolumn{10}{|c|}{ Healthy dietary pattern } & \multicolumn{10}{|c|}{ Western dietary pattern } \\
\hline & \multicolumn{2}{|c|}{ Q1 } & \multicolumn{2}{|c|}{ Q2 } & \multicolumn{2}{|c|}{ Q3 } & \multicolumn{2}{|c|}{ Q4 } & \multirow[b]{2}{*}{$\begin{array}{l}P \text { for } \\
\text { trend }\end{array}$} & \multirow[b]{2}{*}{$P_{\mathrm{i}}$} & \multicolumn{2}{|c|}{ Q1 } & \multicolumn{2}{|c|}{ Q2 } & \multicolumn{2}{|c|}{ Q3 } & \multicolumn{2}{|c|}{ Q4 } & \multirow[b]{2}{*}{$\begin{array}{l}P \text { for } \\
\text { trend }\end{array}$} & \multirow[b]{2}{*}{$P_{\mathrm{i}}$} \\
\hline & Mean & SEM & Mean & SEM & Mean & SEM & Mean & SEM & & & Mean & SEM & Mean & SEM & Mean & SEM & Mean & SEM & & \\
\hline rs1121980 & & & & & & & & & & 0.50 & & & & & & & & & & 0.03 \\
\hline $\mathrm{CC}$ & 0.90 & 0.41 & 1.53 & 0.39 & $1 \cdot 26$ & 0.39 & 1.73 & 0.41 & 0.40 & & 1.46 & 0.42 & 0.69 & 0.39 & 1.49 & 0.40 & 1.69 & 0.40 & 0.19 & \\
\hline $\mathrm{CT}+\mathrm{TT}$ & 1.73 & 0.32 & 1.50 & 0.30 & $2 \cdot 08$ & 0.30 & 2.06 & 0.32 & 0.36 & & $1 \cdot 19$ & 0.32 & $2 \cdot 29$ & 0.31 & 1.65 & 0.30 & $2 \cdot 16$ & 0.32 & 0.05 & \\
\hline rs1421085 & & & & & & & & & & $0 \cdot 2$ & & & & & & & & & & 0.007 \\
\hline $\mathrm{TT}$ & 0.95 & 0.40 & 1.66 & 0.38 & $1 \cdot 12$ & 0.38 & 1.68 & 0.40 & 0.30 & & 1.62 & 0.41 & 1.77 & 0.38 & 1.64 & 0.39 & 1.36 & 0.38 & 0.15 & \\
\hline $\mathrm{CT}+\mathrm{CC}$ & 1.75 & 0.32 & 1.40 & 0.31 & $2 \cdot 17$ & 0.30 & $2 \cdot 08$ & 0.32 & 0.43 & & 1.08 & 0.32 & $2 \cdot 28$ & 0.32 & 1.65 & 0.30 & $2 \cdot 41$ & 0.33 & 0.01 & \\
\hline rs9939973 & & & & & & & & & & 0.38 & & & & & & & & & & 0.06 \\
\hline GG & 0.88 & 0.40 & 54 & 0.39 & $\cdot 17$ & 0.39 & $1 \cdot 70$ & 0.41 & 0.4 & & 1.40 & 0.41 & 0.7 & 0.38 & 1.5 & 0.39 & 1.6 & 0.4 & 0 & \\
\hline$A G+A A$ & 1.72 & 0.32 & 1.45 & 0.30 & $2 \cdot 11$ & 0.30 & 2.05 & 0.32 & 0.30 & & 1.21 & 0.32 & $2 \cdot 25$ & 0.31 & 1.68 & 0.30 & $2 \cdot 18$ & 0.32 & 0.14 & \\
\hline rs8050136 & & & & & & & & & & 0.06 & & & & & & & & & & 0.005 \\
\hline CC & 0.93 & 0.39 & 1.73 & 0.36 & $1 \cdot 24$ & 0.36 & 1.67 & 0.38 & 0.38 & & 1.52 & 0.39 & 0.72 & 0.37 & 1.55 & 0.37 & 1.75 & 0.37 & 0.72 & \\
\hline $\mathrm{AC}+\mathrm{AA}$ & 1.730 & 0.33 & 1.337 & 0.32 & $2 \cdot 16$ & 0.31 & $2 \cdot 12$ & 0.33 & 0.29 & & 1.09 & 0.33 & $2 \cdot 38$ & 0.32 & 1.73 & 0.31 & $2 \cdot 14$ & 0.34 & 0.02 & \\
\hline rs1781799 & & & & & & & & & & 0.19 & & & & & & & & & & 0.02 \\
\hline $\mathrm{TT}$ & 0.95 & 0.38 & 1.69 & 0.36 & $1 \cdot 27$ & 0.36 & 1.63 & 0.38 & 0.37 & & 1.50 & 0.39 & 0.76 & 0.37 & 1.53 & 0.37 & 1.69 & 0.36 & 0.12 & \\
\hline $\mathrm{TG}+\mathrm{GG}$ & 1.75 & 0.33 & 1.32 & 0.32 & $2 \cdot 14$ & 0.31 & 2.09 & 0.33 & 0.38 & & $1 \cdot 10$ & 0.33 & 2.33 & 0.32 & 1.66 & 0.31 & $2 \cdot 20$ & 0.34 & 0.04 & \\
\hline rs3751812 & & & & & & & & & & 0.14 & & & & & & & & & & 0.03 \\
\hline GG & 0.88 & 0.39 & $1 \cdot 80$ & 0.37 & $1 \cdot 19$ & 0.36 & 1.62 & 0.39 & 0.41 & & 1.46 & 0.40 & 0.83 & 0.37 & 1.49 & 0.38 & 1.65 & 0.37 & 0.83 & \\
\hline$T G+T T$ & 1.75 & 0.34 & 1.40 & 0.32 & $2 \cdot 18$ & 0.32 & $2 \cdot 11$ & 0.34 & 0.30 & & 1.05 & 0.34 & $2 \cdot 35$ & 0.33 & 1.73 & 0.32 & $2 \cdot 32$ & 0.35 & 0.04 & \\
\hline GRS & & & & & & & & & & & & & & & & & & & & 0.01 \\
\hline $\mathrm{GRS}<6$ & 23 & 0.39 & & & 1.46 & 0.37 & 1.7 & & & 0.28 & & & & 0.37 & 1.7 & & $1 \cdot \varepsilon$ & & 0.2 & \\
\hline $\mathrm{GRS} \geq 6$ & 1.90 & 0.34 & 1.41 & 0.33 & $2 \cdot 20$ & 0.32 & 1.94 & 0.34 & 0.81 & & 1.04 & 0.34 & $2 \cdot 82$ & 0.33 & $1 \cdot 70$ & 0.32 & $2 \cdot 26$ & 0.36 & 0.04 & \\
\hline
\end{tabular}

$P_{\mathrm{i}}, P_{\text {interaction. }}$

* BMI changes were calculated by subtracting the BMI at baseline from their measurements over a mean of 3-year follow-up; increases in BMI were defined if BMI changes were positive or $>0$. Participants were jointly classified (eight groups) according to quartiles of dietary pattern scores and dominant model of FTO polymorphism genotypes or GRS $\geq$ median and <median. Models were adjusted for age, sex, baseline BMI, educational level, smoking status, physical activity and energy intake.

Table 5. Changes in waist circumference $(\mathrm{cm})$ according to quartiles of dietary pattern scores $(\mathrm{Q})$ by fat mass and obesity-associated (FTO) genotypes and genetic risk score (GRS) in adult participants of the Tehran Lipid and Glucose Study*

(Mean values with their standard errors)

\begin{tabular}{|c|c|c|c|c|c|c|c|c|c|c|c|c|c|c|c|c|c|c|c|c|}
\hline & \multicolumn{10}{|c|}{ Healthy dietary pattern } & \multicolumn{10}{|c|}{ Western dietary pattern } \\
\hline & \multicolumn{2}{|c|}{ Q1 } & \multicolumn{2}{|c|}{ Q2 } & \multicolumn{2}{|c|}{ Q3 } & \multicolumn{2}{|c|}{ Q4 } & \multirow{2}{*}{$\begin{array}{l}P \text { for } \\
\text { trend }\end{array}$} & \multirow[b]{2}{*}{$P_{\mathrm{i}}$} & \multicolumn{2}{|c|}{ Q1 } & \multicolumn{2}{|c|}{ Q2 } & \multicolumn{2}{|c|}{ Q3 } & \multicolumn{2}{|c|}{ Q4 } & \multirow{2}{*}{$\begin{array}{l}P \text { for } \\
\text { trend }\end{array}$} & \multirow[b]{2}{*}{$P_{\mathrm{i}}$} \\
\hline & Mean & SEM & Mean & SEM & Mean & SEM & Mean & SEM & & & Mean & SEM & Mean & SEM & Mean & SEM & Mean & SEM & & \\
\hline rs1121980 & & & & & & & & & & 0.74 & & & & & & & & & & 0.06 \\
\hline $\mathrm{CC}$ & 0.69 & 0.39 & 0.59 & 0.38 & 0.71 & 0.37 & 0.69 & 0.39 & 0.84 & & 1.06 & 0.40 & 0.62 & 0.37 & 1.01 & 0.38 & 0.74 & 0.38 & 0.28 & \\
\hline FTT & & 0.31 & 0.57 & 0.29 & $1 \cdot 12$ & 0.29 & 0.75 & 0.31 & 0.26 & & & 0.31 & 0.95 & 0.30 & 0.53 & 0.29 & 0.85 & 0.31 & 0.17 & \\
\hline rs1421085 & & & & & & & & & & 0.24 & & & & & & & & & & 0.04 \\
\hline TT & 0.93 & 0.38 & 0.75 & 0.36 & 0.57 & 0.37 & 0.62 & 0.39 & 0.97 & & 1.07 & 0.39 & 0.39 & 0.36 & $1 \cdot 19$ & 0.37 & 0.64 & 0.37 & 0.35 & \\
\hline $\mathrm{CT}+\mathrm{CC}$ & 0.35 & 0.31 & 0.53 & 0.30 & $1 \cdot 21$ & 0.29 & 0.80 & 0.31 & 0.20 & & 0.53 & 0.31 & 0.94 & 0.30 & 0.53 & 0.29 & 0.94 & 0.32 & 0.08 & \\
\hline rs9939973 & & & & & & & & & & 0.51 & & & & & & & & & & 0.08 \\
\hline GG & 78 & 0.39 & 65 & 0.38 & 0.66 & 0.37 & 0.60 & 0.39 & 0.95 & & 0.99 & 40 & 0.00 & 0.37 & 1.08 & 0.38 & 68 & .38 & 0.26 & \\
\hline$A G+A A$ & 0.32 & 0.31 & 0.56 & 0.29 & 1.13 & 0.29 & 0.77 & 0.32 & 0.20 & & 0.57 & 0.31 & 0.89 & 0.30 & 0.52 & 0.29 & 0.84 & 0.31 & 0.19 & \\
\hline rs 8050 & & & & & & & & & & 0.30 & & & & & & & & & & 0.15 \\
\hline $\mathrm{CC}$ & 0.78 & 0.37 & 0.80 & 0.35 & 0.58 & 0.35 & 0.54 & 0.37 & 0.80 & & 1.01 & 0.38 & 0.06 & 0.35 & 0.99 & 0.36 & 0.67 & 0.35 & 0.11 & \\
\hline$A C+A A$ & 0.27 & 0.32 & 0.46 & 0.30 & 1.25 & 0.30 & 0.86 & 0.32 & 0.18 & & 0.48 & 0.32 & 0.97 & 0.31 & 0.58 & 0.30 & 0.85 & 0.33 & 0.29 & \\
\hline rs1781 & & & & & & & & & & 0.20 & & & & & & & & & & 0.13 \\
\hline $\mathrm{TT}$ & 83 & 0.37 & .77 & 0.35 & 0.62 & 0.35 & 0.53 & 0.37 & 0.83 & & & & & 0.35 & & & & 0.35 & 0.1 & \\
\hline $\mathrm{TG}+\mathrm{GG}$ & 0.29 & 0.31 & 0.45 & 0.30 & 1.25 & 0.30 & 0.86 & 0.32 & 0.22 & & 0.53 & 0.31 & 0.91 & 0.31 & 0.55 & 0.30 & 0.89 & 0.33 & 0.42 & \\
\hline rs3751812 & & & & & & & & & & 0.24 & & & & & & & & & & 0.04 \\
\hline GG & 0.77 & 0.37 & 0.90 & 0.35 & 0.51 & 0.35 & 0.58 & 0.37 & 0.84 & & & & 0.06 & 0.35 & & & & & & \\
\hline $\mathrm{TG}+\mathrm{TT}$ & 0.29 & 0.34 & 0.49 & 0.31 & 1.30 & 0.31 & 0.87 & 0.32 & 0,15 & & 0.46 & 0.32 & 0.97 & 0.31 & 0.55 & 0.30 & 1.01 & 0.33 & 0.06 & \\
\hline GRS & & & & & & & & & & 0.19 & & & & & & & & & & 0.0 \\
\hline $\mathrm{GRS}<6$ & 1.07 & 0.38 & 0.86 & 0.36 & 0.71 & 0.35 & 0.64 & 0.37 & 0.69 & & $1 \cdot 1$ & 0.38 & & 0.36 & $1 \cdot 1$ & 0.36 & $0 . \varepsilon$ & 0.36 & 0.49 & \\
\hline $\mathrm{GRS} \geq 6$ & 0.41 & 0.32 & 0.45 & 0.31 & 1.32 & 0.31 & 0.72 & 0.33 & $0 \cdot 13$ & & 0.47 & 0.32 & 1.02 & 0.32 & 0.51 & 0.30 & 0.95 & 0.34 & 0.12 & \\
\hline
\end{tabular}

$P_{\mathrm{i}}, P_{\text {interaction. }}$

* Waist circumference changes were calculated by subtracting the waist circumference at baseline from their measurements over a mean of 3-year follow-up; increases in waist circumference were defined if their changes were positive or $>0$. Participants were jointly classified (eight groups) according to quartiles of dietary pattern scores and dominant model of FTO polymorphism genotypes or GRS $\geq$ median and $<$ median. Models were adjusted for age, sex, baseline waist circumference, educational level, smoking status, physical activity and energy intake. 
increasing quartiles of the Western dietary pattern score, compared with the low GRS group (WC change; Q1: 0.47 (sE 0.32) $v$. Q4: 0.95 (sE 0.34)) $\left(P_{\text {interaction }}<0.05\right)$. On analysing healthy dietary pattern score, there was no significant interaction with GRS on WC change.

\section{Discussion}

Two major dietary patterns were identified in our study, the healthy and the Western. The Western or unhealthy dietary pattern could modify the association of FTO SNP or their GRS with general and abdominal obesity in a prospective cohort study of a Tehranian population. Higher adherence to Western dietary pattern was associated with about 2-fold increase in BMI and WC change among adult subjects having FTO risk alleles. Also the healthy dietary pattern did not have any interaction with FTO SNP singly or in combination in relation to changes in obesity phenotypes.

This is the first study to examine available FTO SNP and posteriori-dietary pattern (extracted through principal component analysis) interaction in relation to changes in obesity phenotypes in a Middle Eastern population. Defining dietary patterns considers the combined effect of food groups and nutrients, which clearly explains details of dietary intake ${ }^{(32)}$. In the present study sweetened fruit juices and salty snacks were included in the healthy dietary pattern; these findings indicate that our participants had not considered the added sugar and salt content of these foods and they consumed these foods in the same frequency with the healthy foods. This point should be emphasised in nutrition education planning.

Previous studies often focused on the effect of interaction of different dietary macronutrients with FTO polymorphisms in relation to obesity ${ }^{(33,34)}$. Dietary protein intake attenuated the effect of FTO risk variant rs1558902 in relation to BMI or $\mathrm{WC}^{(35)}$; however, a meta-analysis found no interaction between FTO SNP rs9939609 and dietary protein, carbohydrate, fat or energy intake in relation to $\mathrm{BMI}^{(36)}$. Higher intake of dietary fibre attenuated the association of FTO SNP with general obesity particularly in subjects with high GRS ${ }^{(37)}$. Previous studies showed that some food groups such as sugar-sweetened beverages ${ }^{(30)}$ and fried foods ${ }^{(29)}$ (top-ranked food groups in the Western dietary pattern) had interaction with FTO genotypes rs9939609 in relation to risk of obesity, results in line with our findings. In the HUNT study, an interaction effect of FTO rs9939609 with artificially sweetened beverages in relation to obesity was found only in men aged 20-60 years, indicating that the interaction term may be affected under some conditions such as age groups and $\operatorname{sex}^{(38)}$.

Moreover, the GRS of combined SNP is established to describe more variations in BMI and gaining statistical power in comparison with single $\mathrm{SNP}^{(39)}$. The linkage disequilibrium between rs9939609 and the three SNP (rs8050136, rs3751812, rs17817449) is high ( $\left.r^{2} 1\right)$ based on data from South and East Asians ${ }^{(31,40)}$; therefore the rs9939609 was not entered in GRS calculation and analysis. Also due to limited resources available, the most reproducible associated SNP with obesity and dietary intake were selected.

Unlike our findings, the Wang et al. study described that higher adherence to predefined dietary patterns, including the alternate healthy eating index-2010 and the dietary approach to stop hypertension diet, could decrease the association of genetic predisposition score with weight gain; also improving diet quality had a beneficial effect on weight management particularly in people with high genetic risk for obesity in two cohorts from the USA ${ }^{(41)}$. The alternate healthy eating index2010 score was scaled based on eleven food groups and nutrients with an extremely wide range and high sensitivity for differentiating between individuals with different dietary patterns.

A higher adherence to the Mediterranean dietary pattern was associated with lower obesity risk in subjects with high GRS, compared with individuals with low GRS and lower adherence to the Mediterranean dietary pattern ${ }^{(42)}$. In our study, salty snacks and sweetened fruit juices were included in the healthy dietary pattern which indicated that participants consumed these food groups along with other healthy food items with a median correlation. Also subjects in the upper quartiles of the healthy dietary pattern score had higher energy intake than individuals in lower quartiles due to overconsumption of foods; this observation can be explained by the fact that total energy intake is more relevant than dietary fat intake, and people merely replace energy from fat with energy from carbohydrates. Also, variations in food group intake and overconsumption of foods may restrict the sensitivity of the healthy dietary pattern and contribute to the lack of the interaction term between this pattern and FTO GRS in relation to obesity traits.

In a meta-analysis of weight loss trial, individuals carrying the homozygous risk allele of FTO variant rs9939609 (or proxy SNP) had greater weight loss than non-carriers after diet/lifestyle trials; the effect size was greater with diet intervention than other lifestyle interventions, considering adjustment for baseline $\mathrm{BMI}^{(43)}$. In another meta-analysis of 9563 participants of eight randomised clinical trials, subjects carrying the FTO genotypes respond equally to adiposity measure after weight loss interventions ${ }^{(44)}$.

Higher adherence to the Western dietary pattern was inversely associated with obesity at baseline, in spite of more increments in BMI and WC after follow-up years, in contrary to subjects with higher adherence to the healthy dietary pattern; this may suggest that obese subjects had a lower rate of increase in BMI than normal-weight subjects ${ }^{(45)}$.

The prospective design and broad adjustment for potential confounding factors are the strengths of our study. Its results come from an approximately homogeneous population. Data measurements were not self-reported and gathered by trained questioners.

The present study has limitations that need to be considered. There are confounding factors such as mental health and sleeping patterns that were not entered in the model. Lack of availability of a complete and standardised Iranian FCT is another limitation of our study. However, nutrient contents of specific foods are relatively consistent, and deviations may not be large enough to alter dietary intake. Also the estimation of usual nutrient intake from FFQ during the previous year reduces much error due to variations in nutrient compositions. Assessing dietary intake at only one time point is the other limitation of the present study.

Extracting dietary patterns through principal component analysis is subjective ${ }^{(32)}$, although eigenvalues, scree-plots and 
interpretability lead the researcher to determine the best factor solution.

Considering the diminished effect of FTO minor alleles on obesity at older ages ${ }^{(33,38)}$, it is suggested to design future studies with large enough sample sizes to cover statistical power for analysing the effect of interaction of FTO GRS with dietary factors regarding obesity traits in an age- and sex-dependent manner.

\section{Conclusion}

Our findings demonstrated that the unhealthy or Western dietary pattern could increase the association of genetic susceptibility of FTO SNP with BMI or WC change. These results support dietary recommendations to reduce the consumption of unhealthy dietary pattern, including sweetened beverages, sweets, highfat red meat, solid fats, refined grains, fast foods, salty snacks and high-fat dairy products for the prevention of obesity, namely, in subjects with more genetic predisposition to obesity.

\section{Acknowledgements}

The authors wish to acknowledge Ms Niloofar Shiva for critical editing of English grammar and syntax of the manuscript.

The present study was supported by the Research Institute for Endocrine Sciences, Shahid Beheshti University of Medical Sciences, Tehran, Iran (grant no. 840).

Authors' contributions are as follows: F. H. E., G. K. and M. S. D. designed the study, analysed and interpreted the data and drafted the manuscript; M. S. D. and P. M. supervised the study, F. A. revised the manuscript for important intellectual content and final approval of the version to be published. All authors approved the final version of manuscript.

The authors declare that they have no conflict of interest.

\section{References}

1. World Health Organization (2000) Obesity: Preventing and Managing the Global Epidemic. Report of a WHO Consultation. World Health Organization Technical Report Series no. 894. Geneva: WHO.

2. Gill T (2006) Epidemiology and health impact of obesity: an Asia Pacific perspective. Asia Pac J Clin Nutr 15, 3-14.

3. Aranceta J, Moreno B, Moya M, et al. (2009) Prevention of overweight and obesity from a public health perspective. Nutr Rev 67, Suppl. 1, S83-S88.

4. Ghalandari H, Hosseini-Esfahani F \& Mirmiran P (2015) The association of polymorphisms in leptin/leptin receptor genes and ghrelin/ghrelin receptor genes with overweight/obesity and the related metabolic disturbances: a review. Int $J$ Endocrinol Metab 13, e19073.

5. Smemo S, Tena JJ, Kim KH, et al. (2014) Obesity-associated variants within FTO form long-range functional connections with IRX3. Nature 507, 371-375.

6. Corella D, Ortega-Azorin C, Sorli JV, et al. (2012) Statistical and biological gene-lifestyle interactions of MC4R and FTO with diet and physical activity on obesity: new effects on alcohol consumption. PLOS ONE 7, e52344.

7. Hardy R, Wills AK, Wong A, et al. (2010) Life course variations in the associations between FTO and MC4R gene variants and body size. Hum Mol Genet 19, 545-552.
8. Labayen I, Ruiz JR, Huybrechts I, et al. (2016) Dietary fat intake modifies the influence of the FTO rs9939609 polymorphism on adiposity in adolescents: the HELENA cross-sectional study. Nutr Metab Cardiovasc Dis 26, 937-943.

9. Moleres A, Ochoa MC, Rendo-Urteaga T, et al. (2012) Dietary fatty acid distribution modifies obesity risk linked to the rs9939609 polymorphism of the fat mass and obesityassociated gene in a Spanish case-control study of children. Br J Nutr 107, 533-538.

10. Nicklas TA, Baranowski T, Cullen KW, et al. (2001) Eating patterns, dietary quality and obesity. J Am Coll Nutr 20, 599-608.

11. Togo P, Osler M, Sorensen TI, et al. (2004) A longitudinal study of food intake patterns and obesity in adult Danish men and women. Int J Obes Relat Metab Disord 28, 583-593.

12. Azizi F, Rahmani M, Emami H, et al. (2002) Cardiovascular risk factors in an Iranian urban population: Tehran Lipid and Glucose Study (phase 1). Soz Praventivmed 47, 408-426.

13. Azizi F, Ghanbarian A, Momenan AA, et al. (2009) Prevention of non-communicable disease in a population in nutrition transition: Tehran Lipid and Glucose Study phase II. Trials 10, 5.

14. Esfahani FH, Asghari G, Mirmiran P, et al. (2010) Reproducibility and relative validity of food group intake in a food frequency questionnaire developed for the Tehran Lipid and Glucose Study. J Epidemiol 20, 150-158.

15. Mirmiran P, Esfahani FH, Mehrabi Y, et al. (2010) Reliability and relative validity of an FFQ for nutrients in the Tehran Lipid and Glucose Study. Public Health Nutr 13, 654-662.

16. Azar M, \&Sarkisian E., (1980) Food Composition Table of Iran. Tehran: National Nutrition and Food Research Institute, Shahid Beheshti University Press.

17. US Department of Agriculture (2014) USDA Food Composition Databases. Washington, DC: United States Department of Agriculture. https://ndb.nal.usda.gov/ndb/ (accessed January 2015).

18. Momenan AA, Delshad M, Sarbazi N, et al. (2012) Reliability and validity of the Modifiable Activity Questionnaire (MAQ) in an Iranian urban adult population. Arch Iran Med 15, 279-282.

19. European Bioinformatics Institute (2017) GWAS Catalog. The NHGRI-EBI Catalog of Published Genome-Wide Association Studies. https://www.ebi.ac.uk/gwas/ (accessed September 2017).

20. National Center for Biotechnology Information (2017) PheGenI Phenotype-Genotype Integrator. https://www.ncbi.nlm.nih. gov/gap/phegeni (accessed January 2017).

21. Lee HJ, Kim IK, Kang JH, et al. (2010) Effects of common FTO gene variants associated with BMI on dietary intake and physical activity in Koreans. Clin Chim Acta 411, 1716-1722.

22. McCaffery JM, Papandonatos GD, Peter I, et al. (2012) Obesity susceptibility loci and dietary intake in the Look AHEAD Trial. Am J Clin Nutr 95, 1477-1486.

23. Moore SC, Gunter MJ, Daniel CR, et al. (2012) Common genetic variants and central adiposity among Asian-Indians. Obesity (Silver Spring) 20, 1902-1908.

24. Peng S, Zhu Y, Xu F, et al. (2011) FTO gene polymorphisms and obesity risk: a meta-analysis. BMC Med $\mathbf{9}, 71$.

25. Tanaka T, Ngwa JS, van Rooij FJ, et al. (2013) Genome-wide meta-analysis of observational studies shows common genetic variants associated with macronutrient intake. Am J Clin Nutr 97, 1395-1402.

26. Wing MR, Ziegler J, Langefeld CD, et al. (2009) Analysis of FTO gene variants with measures of obesity and glucose homeostasis in the IRAS Family Study. Hum Genet 125, 615-626.

27. Miller SA, Dykes DD \& Polesky HF (1988) A simple salting out procedure for extracting DNA from human nucleated cells. Nucleic Acids Res 16, 1215. 
28. Daneshpour MS, Fallah MS, Sedaghati-Khayat B, et al. (2017) Rationale and design of a genetic study on cardiometabolic risk factors: protocol for the Tehran Cardiometabolic Genetic Study (TCGS). JMIR Res Protoc 6, e28.

29. Qi Q, Chu AY, Kang JH, et al. (2014) Fried food consumption, genetic risk, and body mass index: gene-diet interaction analysis in three US cohort studies. BMJ 348, g1610.

30. Qi Q, Chu AY, Kang JH, et al. (2012) Sugar-sweetened beverages and genetic risk of obesity. $N$ Engl J Med 367, 1387-1396.

31. Alharbi KK, Richardson TG, Khan IA, et al. (2014) Influence of adiposity-related genetic markers in a population of Saudi Arabians where other variables influencing obesity may be reduced. Dis Markers 2014, 758232

32. Hosseini-Esfahani F, Djazaieri SA, Mirmiran P, et al. (2012) Which food patterns are predictors of obesity in Tehranian adults? J Nutr Educ Behav 44, 564-573.

33. Corella D, Arnett DK, Tucker KL, et al. (2011) A high intake of saturated fatty acids strengthens the association between the fat mass and obesity-associated gene and BMI. J Nutr 141, 2219-2225.

34. Lappalainen T, Lindstrom J, Paananen J, et al. (2012) Association of the fat mass and obesity-associated (FTO) gene variant (rs9939609) with dietary intake in the Finnish Diabetes Prevention Study. Br J Nutr 108, 1859-1865.

35. Merritt DC, Jamnik J \& El-Sohemy A (2018) FTO genotype, dietary protein intake, and body weight in a multiethnic population of young adults: a cross-sectional study. Genes Nutr 13, 4 .

36. Qi Q, Kilpelainen TO, Downer MK, et al. (2014) FTO genetic variants, dietary intake and body mass index: insights from 177,330 individuals. Hum Mol Genet 23, 6961-6972.

37. Hosseini-Esfahani F, Koochakpoor G, Daneshpour MS, et al. (2017) The interaction of fat mass and obesity associated gene polymorphisms and dietary fiber intake in relation to obesity phenotypes. Sci Rep 7, 18057

38. Bjornland T, Langaas M, Grill V, et al. (2017) Assessing gene-environment interaction effects of FTO, MC4R and lifestyle factors on obesity using an extreme phenotype sampling design: results from the HUNT study. PLOS ONE $\mathbf{1 2}$, e0175071.

39. Rask-Andersen M, Karlsson T, Ek WE, et al. (2017) Gene-environment interaction study for BMI reveals interactions between genetic factors and physical activity, alcohol consumption and socioeconomic status. PLoS Genet 13, e1006977.

40. Li H, Kilpelainen TO, Liu C, et al. (2012) Association of genetic variation in FTO with risk of obesity and type 2 diabetes with data from 96,551 East and South Asians. Diabetologia 55 , 981-995.

41. Wang T, Heianza Y, Sun D, et al. (2018) Improving adherence to healthy dietary patterns, genetic risk, and long term weight gain: gene-diet interaction analysis in two prospective cohort studies. BMJ 360, j5644.

42. Hosseini-Esfahani F, Koochakpoor G, Daneshpour MS, et al. (2017) Mediterranean dietary pattern adherence modify the association between FTO genetic variations and obesity phenotypes. Sci Rep 9, E1064.

43. Xiang L, Wu H, Pan A, et al. (2016) FTO genotype and weight loss in diet and lifestyle interventions: a systematic review and meta-analysis. Am J Clin Nutr 103, 1162-1170.

44. Livingstone KM, Celis-Morales C, Papandonatos GD, et al. (2016) FTO genotype and weight loss: systematic review and meta-analysis of 9563 individual participant data from eight randomised controlled trials. BMJ 354, i4707.

45. Akbarzadeh M, Moghimbeigi A, Mahjub H, et al. (2016) Trajectories of change in obesity among tehranian families: multilevel latent growth curve modeling. Int J Family Med 2016, 2639624.

(1)

